\title{
PRIME GENERALIZED ALTERNATIVE RINGS I WITH NONTRIVIAL IDEMPOTENT
}

\author{
HARRY F. SMITH
}

\begin{abstract}
A generalized alternative ring $I$ is a nonassociative ring $R$ in which the identities $(w x, y, z)+(w, x,[y, z])-w(x, y, z)-$ $(w, y, z) x ; \quad([w, x], y, z)+(w, x, y z)-y(w, x, z)-(w, x, y) z ;$ and $(x, x, x)$ are identically zero. It is demonstrated here that if $R$ is a ring of this type with characteristic different from two and three, then $R$ semiprime with idempotent $e$ implies that $R$ has a Peirce decomposition relative to $e$. Furthermore, if $R$ is prime and $e \neq 0,1$; then $R$ must be alternative.
\end{abstract}

1. Introduction. Let $R$ be a nonassociative ring. As is customary, for $x, y, z \in R$ we denote by $(x, y, z)$ the associator $(x, y, z)=(x y) z-x(y z)$ and by $[x, y]$ the commutator $[x, y]=x y-y x . R$ is called power-associative if for every $x \in R$ the subring generated by $x$ is associative.

In [2] Kleinfeld defines a generalized alternative ring $I$ to be a nonassociative ring $R$ such that for all $w, x, y, z \in R$ the following identities are satisfied:

(1) $(w x, y, z)+(w, x,[y, z])=w(x, y, z)+(w, y, z) x$,

(2) $([w, x], y, z)+(w, x, y z)=y(w, x, z)+(w, x, y) z$,

(3) $(x, x, x)=0$.

It is easily verified that a ring of this type is power-associative.

Consider $R$ to be a power-associative ring of characteristic different from two and define $x \circ y=\frac{1}{2}(x y+y x)$ for $x, y \in R$. If $R$ contains an idempotent $e$, then Albert has shown in [1] that $R=R_{1}(e)+R_{1 / 2}(e)+R_{0}(e)$ where $R_{i}(e)=\{x \in R: x \circ e=i x\}$. In fact, $e x=x=x e$ for $x \in R_{1}(e)$ and $e x=$ $0=x e$ for $x \in R_{0}(e)$. This decomposition of $R$ is known as the Albert decomposition. Throughout this work we will denote $R_{i}(e)$ by just $R_{i}$.

Suppose now one also has $(R, e, e)=(e, R, e)=(e, e, R)=0$. If, as in the associative case, one takes $x=e x e+(e x-e x e)+(x e-e x e)+$ $(x-e x-x e+e x e)$, one sees that $R=R_{11}+R_{10}+R_{01}+R_{00}$ where $R_{i j}=$ $\{x \in R: e x=i x, x e=j x\}$. This further decomposition of $R$ is referred to as the Peirce decomposition.

Presented to the Society, September 1, 1971 under the title On generalized alternative rings I and II; received by the editors October 6, 1972.

AMS (MOS) subject classifications (1970). Primary 17A30.

Key words and phrases. Generalized alternative ring I, semiprime ring, Peirce decomposition, prime ring, alternative ring.

(c) American Mathematical Society 1973 
A nonassociative ring $R$ is said to be semiprime if $R$ contains no nonzero ideal $I$ such that $I^{2}=0$. If, in addition, $R$ contains no nonzero ideals $I$ and $H$ such that $I H=0$, then $R$ is said to be prime.

2. Preliminaries. Henceforth we assume $R$ to be a generalized alternative ring $I$ with characteristic different from two and three. Under the additional assumption that $R$ contains an idempotent $e$, we will make use of the following results established by Kleinfeld in [2]:

(i) $(e, R, e)=0$.

(ii) In the Albert decomposition, $R_{1 / 2} R_{i} \subseteq R_{1 / 2}$ and $R_{i} R_{1 / 2} \subseteq R_{1 / 2}$ for $i=0,1$. Furthermore, $B=\left\{x \in R_{1 / 2}: x R \subseteq R_{1 / 2}, R x \subseteq R_{1 / 2}\right\}$ is an ideal of $R$ such that $(e, e, R) \subseteq B$ and every element of $B$ squares to zero.

(iii) If $R$ permits a Peirce decomposition, then for $i, j, k, l=0$ or 1 , $R_{i j} R_{k l}=0$ when $j \neq k$ and $R_{i j} R_{j l} \subseteq R_{i l}$, with two exceptions, namely $R_{01} R_{01} \subseteq R_{10}$ and $R_{10} R_{10} \subseteq R_{01}$. Furthermore, if $\left(R_{01}, R_{11}, R_{11}\right)=$ $\left(R_{11}, \quad R_{11}, \quad R_{10}\right)=\left(\begin{array}{llll}R_{10}, & R_{00}, & R_{00}\end{array}\right)=\left(\begin{array}{lll}R_{00}, & R_{00}, & R_{01}\end{array}\right)=0$, then $I_{i}=$ $\sum\left(R_{i i}, R_{i i}, R_{i i}\right)+\sum\left(R_{i i}, R_{i i}, R_{i i}\right) R_{i i}$ for $i=0,1$ are ideals of $R$.

The following identities will also be necessary for our calculations:

(4) $(w x, y, z)-(w, x y, z)+(w, x, y z)=w(x, y, z)+(w, x, y) z$,

(5) $(w, x y, z)-(w, x, z y)+(w, x, y) z-(w, y, z) x=0$,

(6) $(w, x y, z)-(x w, y, z)+w(x, y, z)-y(w, x, z)=0$,

(7) $(x, x, y z)=y(x, x, z)+(x, x, y) z$.

A straightforward verification shows that (4), known as the Teichmüller identity, holds for all $w, x, y, z$ in any nonassociative ring. Identity (5) is obtained by subtracting (4) from (1), while (6) is obtained by subtracting (4) from (2). Identity (7) follows from letting $w=x$ in identity (2).

\section{Semiprime and prime rings.}

THEOREM 1. Let $R$ be a generalized alternative ring $I$ with characteristic different from two and three. If $R$ contains an idempotent $e$, then $I=$ $(e, e, R)$ is an ideal of $R$ such that $I^{2}=0$. Furthermore, $[I, R]=0$ and $R_{1 / 2} I=\left(R_{1 / 2}\right)^{2} I=0$.

Proof. By (ii), $B=\left\{x \in R_{1 / 2}: x R \subseteq R_{1 / 2}, R x \subseteq R_{1 / 2}\right\}$ is an ideal of $R$ such that $(e, e, R) \subseteq B$ and every element of $B$ squares to zero. Let $K=$ $(e, e, B) \subseteq B$. We first show that $K$ is an ideal of $R$ such that $0=K^{2}=$ $[K, R]=R_{1 / 2} K=\left(R_{1 / 2}\right)^{2} K$.

We begin by making the following observations. Let $(e, y, z)=$ $a_{1}+a_{1 / 2}+a_{0}$ and $(e, e,[y, z])=b_{1 / 2}$ where $a_{i} \in R_{i}$ for $i=0, \frac{1}{2}, 1$ and $b_{1 / 2} \in R_{1 / 2}$. Then (1) yields $(e, y, z)+(e, e,[y, z])=e(e, y, z)+(e, y, z) e$ or $a_{1}+a_{1 / 2}+a_{0}+b_{1 / 2}=a_{1}+e a_{1 / 2}+a_{1}+a_{1 / 2} e$, whence $a_{1}=a_{0}=b_{1 / 2}=0$. Since 
from [1] we know $y, z \in R_{1 / 2}$ implies $y \circ z \in R_{1}+R_{0}$, we also have $0=(e, e, y z+z y)+(e, e, y z-z y)=2(e, e, y z)$ or $(e, e, y z)=0$ if $y, z \in R_{1 / 2}$.

Now if we let $w=x=z$ in (2) and then apply (3), we have $(z, z, y z)=$ $(z, z, y) z$. If we then let $w=x=y$ in (5) and apply (3), we have $\left(y, y^{2}, z\right)=$ $2(y, y, z) y$. Taking $y=e$ and $z=x$, this gives $(e, e, x)=2(e, e, x) e$. Let $b=(e, e, x)$. Then $b=2 b e$ and, since $b=e b+b e$, this implies $e b=\frac{1}{2} b=b e$. In particular, we have $e k=\frac{1}{2} k=k e$ for every $k \in K$. Now taking $k, k^{\prime} \in$ $K \subseteq R_{1 / 2}$ and using our last observation above together with (7), we have $0=\left(e, e, k k^{\prime}\right)=k\left(e, e, k^{\prime}\right)+(e, e, k) k^{\prime}=\frac{1}{4} k k^{\prime}+\frac{1}{4} k k^{\prime}=\frac{1}{2} k k^{\prime}$ or $K^{2}=0$.

We next show $K$ to be an ideal of $R$. That $K$ is additive is clear. Let $b \in B, k \in K$. Since $B \subseteq R_{1 / 2}$ implies $\left(e, e, B^{2}\right)=0$, using (7) and the fact that $K^{2}=0$ we have $0=(e, e, b k)=b(e, e, k)+(e, e, b) k=b(e, e, k)=\frac{1}{4} b k$ or $B K=0$. Since $b, b^{\prime} \in B$ implies $\left(b+b^{\prime}\right)^{2}=0$ or $b b^{\prime}=-b^{\prime} b$, it follows too that $K B=0$. Let $x_{i} \in R_{i}$ for $i=0, \frac{1}{2}, 1$. Noting that previous calculations show if $b^{\prime}=\left(e, e, x_{1 / 2}\right)$, then $\left(e, e, 4 b^{\prime}\right)=b^{\prime}=\left(e, e, x_{1 / 2}\right)$, we now use (7), the fact that $B K=0=K B$, and the fact that $B$ is an ideal of $R$ to compute as follows:

$$
\begin{aligned}
(e, e, b) x_{i} & =\left(e, e, b x_{i}\right)-b\left(e, e, x_{i}\right)=\left(e, e, b x_{i}\right) \in K \quad \text { for } i=0,1 ; \\
(e, e, b) x_{1 / 2} & =\left(e, e, b x_{1 / 2}\right)-b\left(e, e, x_{1 / 2}\right)=\left(e, e, b x_{1 / 2}\right)-b\left(e, e, 4 b^{\prime}\right) \\
& =\left(e, e, b x_{1 / 2}\right) \in K ; \\
x_{i}(e, e, b) & =\left(e, e, x_{i} b\right)-\left(e, e, x_{i}\right) b=\left(e, e, x_{i} b\right) \in K \quad \text { for } i=1,1 ; \\
x_{1 / 2}(e, e, b) & =\left(e, e, x_{1 / 2} b\right)-\left(e, e, x_{1 / 2}\right) b=\left(e, e, x_{1 / 2} b\right)-\left(e, e, 4 b^{\prime}\right) b \\
& =\left(e, e, x_{1 / 2} b\right) \in K .
\end{aligned}
$$

Thus it follows $K$ is an ideal of $R$.

Suppose now we are given $x \in R$. Let $x=x_{1}+x_{1 / 2}+x_{0}$ where $x_{i} \in R_{i}$ for $i=0, \frac{1}{2}, 1$. Then, using (7) while keeping in mind that $k=(e, e, 4 k)$ for $k \in K$ and $0=(e, e,[y, z])$ for $y, z \in R$, one has for $i=0,1$ that $x_{i} k=$ $x_{i}(e, e, 4 k)+\left(e, e, x_{i}\right)(4 k)=4\left(e, e, x_{i} k\right)=(4 k)\left(e, e, x_{i}\right)+(e, e, 4 k) x_{i}=k x_{i}$. Also keeping in mind that $0=(e, e, y z)$ for $y, z \in R_{1 / 2}$ and $B K=0=K B$, one has $0=\left(e, e, x_{1 / 2} k\right)=x_{1 / 2}(e, e, k)+\left(e, e, x_{1 / 2}\right) k=x_{1 / 2}(e, e, k)=\frac{1}{4} x_{1 / 2} k$ as well as $0=\left(e, e, k x_{1 / 2}\right)=k\left(e, e, x_{1 / 2}\right)+(e, e, k) x_{1 / 2}=\frac{1}{4} k x_{1 / 2}$. Thus $[K, R]=0$ and, in particular, $R_{1 / 2} K=0$.

Next let $x, y \in R_{1 / 2}$ and $k \in K$. Then (1) gives $(x y, e, k)+(x, y,[e, k])=$ $x(y, e, k) \div(x, e, k) y$. But $[K, R]=0$ implies $(x, y,[e, k])=0$, while $K$ an ideal of $R$ with $R_{1 / 2} K=0$ implies $x(y, e, k)=0=(x, e, k) y$. Hence $(x y, e, k)=0$. Let $x y=a_{1}+a_{1 / 2}+a_{0}$ where $a_{i} \in R_{i}$ for $i=0, \frac{1}{2}, 1$. Then $0=$ $(x y, e, k)=[(x y) e] k-\frac{1}{2}(x y) k=\left(a_{1}+a_{1 / 2} e\right) k-\frac{1}{2} a_{1} k-\frac{1}{2} a_{0} k=a_{1} k-\frac{1}{2} a_{1} k-\frac{1}{2} a_{0} k$, using the fact from (ii) that $R_{1 / 2} R_{1} \subseteq R_{1 / 2}$. Thus $a_{1} k=a_{0} k$.

Now from our initial observations we have $(e, x, y) \in R_{1 / 2}$. Hence 
$(e, x, y)=(e x) y-e(x y)=(e x) y-a_{1}-e a_{1 / 2}$ gives $[(e x) y]_{1}=a_{1}$ and $[(e x) y]_{0}=$ 0 . Then $a_{1}+a_{1 / 2}+a_{0}=x y=(e x) y+(x e) y$ implies $[(x e) y]_{1}=0$. Thus (ex) $y \in R_{1}+R_{1 / 2}$ while ( $\left.x e\right) y \in R_{1 / 2}+R_{0}$. Now since from (ii) we know $x e \in R_{1 / 2}$, our previous argument shows $[(x e) y]_{1} k=[(x e) y]_{0} k$. But $[(x e) y]_{1}=0$, so $[(x e) y]_{1} k=0=[(x e) y]_{0} k$. Hence $[(x e) y] k=[(x e) y]_{1} k+$ $[(x e) y]_{1 / 2} k+[(x e) y]_{0} k=0$, since $R_{1 / 2} K=0$. In similar fashion we have $[(e x) y] k=0$. But then $x \in R_{1 / 2}$ gives $(x y) k=[(x e+e x) y] k=[(x e) y] k+$ [(ex)y] $k=0$ or $\left(R_{1 / 2}\right)^{2} K=0$.

Finally, it is clear that $K=(e, e, B) \subseteq(e, e, R)$. But we have also shown above that if $b=\left(e, e, x_{1 / 2}\right)$, then $(e, e, 4 b)=b=\left(e, e, x_{1 / 2}\right)$. Thus $(e, e, R) \subseteq(e, e, 4 B)=K$, that is $K=(e, e, R)$.

COROLLARY. Let $R$ be a generalized alternative ring I with characteristic different from $t$ wo and three. If $R$ contains an idempotent $e$, then $R$ semiprime implies that $R$ has a Peirce decomposition relative to $e$.

Proof. Linearization of (3) gives $(y, x, x)+(x, y, x)+(x, x, y)=0$. By (i), one always has $(e, R, e)=0$. Since, by Theorem $1, I=(e, e, R)$ is an ideal of $R$ such that $I^{2}=0, R$ semiprime now implies in addition that $(e, e, R)=0=(R, e, e)$.

THEOREM 2. Let $R$ be a prime generalized alternative ring $I$ with characteristic different from two and three. If $R$ contains an idempotent $e \neq 0,1$; then $R$ is alternative.

Proof. Since $R$ a prime ring implies that $R$ is semiprime, by the above Corollary $R$ has a Peirce decomposition relative to $e$. Throughout this proof we use the convention that $w_{i j}, x_{i j}, y_{i j}, z_{i j} \in R_{i j}$ for $i, j=0$ or 1 . Then using the multiplication table described by (iii), (5) gives $\left(x_{11}, e y_{11}, z_{10}\right)-$ $\left(x_{11}, e, z_{10} y_{11}\right)+\left(x_{11}, e, y_{11}\right) z_{10}-\left(x_{11}, y_{11}, z_{10}\right) e=0$ and $\left(x_{00}, y_{00} e, z_{01}\right)-$ $\left(x_{00}, y_{00}, z_{01} e\right)+\left(x_{00}, y_{00}, e\right) z_{01}-\left(x_{00}, e, z_{01}\right) y_{00}=0$, that is $\left(R_{11}, R_{11}, R_{10}\right)=$ $0=\left(R_{00}, R_{00}, R_{01}\right)$. Similarly (6) and (iii) yield $\left(x_{01}, y_{11} e, z_{11}\right)-\left(y_{11} x_{01}, e, z_{11}\right)$ $+x_{01}\left(y_{11}, e, z_{11}\right)-e\left(x_{01}, y_{11}, z_{11}\right)=0$ and $\left(x_{10}, e y_{00}, z_{00}\right)-\left(e x_{10}, y_{00}, z_{00}\right)+$ $x_{10}\left(e, y_{00}, z_{00}\right)-y_{00}\left(x_{10}, e, z_{00}\right)=0$, that is $\left(R_{01}, R_{11}, R_{11}\right)=0=\left(R_{10}, R_{00}, R_{00}\right)$. Thus by (iii), $I_{i}=\sum\left(R_{i i}, R_{i i}, R_{i i}\right)+\sum\left(R_{i i}, R_{i i}, R_{i i}\right) R_{i i}$ are ideals for $i=0,1$.

We now use a technique which was first used by Kleinfeld for prime associator-dependent rings. Consider $H=R_{10} R_{01}+R_{10}+R_{01}+R_{01} R_{10}$. Using the multiplication table described by (iii), it follows that to show $H$ is an ideal of $R$ it suffices to verify that $R_{10} R_{01}$ is an ideal of $R_{11}$ and $R_{01} R_{10}$ is an ideal of $R_{00}$. But from (5) and (iii) we have $\left(x_{11}, y_{10} e, z_{01}\right)-$ $\left(x_{11}, y_{10}, z_{01} e\right)+\left(x_{11}, y_{10}, e\right) z_{01}-\left(x_{11}, e, z_{01}\right) y_{10}=0 \quad$ or $\quad\left(R_{11}, R_{10}, R_{01}\right)=0$, while (6) and (iii) yield $\left(x_{10}, e y_{01}, z_{11}\right)-\left(e x_{10}, y_{01}, z_{11}\right)+x_{10}\left(e, y_{01}, z_{11}\right)-$ $y_{01}\left(x_{10}, e, z_{11}\right)=0$ or $\left(R_{10}, R_{01}, R_{11}\right)=0$. Thus $R_{10} R_{01}$ is an ideal of $R_{11}$. 
Analogously one has $\left(R_{00}, R_{01}, R_{10}\right)=0=\left(R_{01}, R_{10}, R_{00}\right)$ or $R_{01} R_{10}$ is an ideal of $R_{00}$.

Now (5) and (iii) give $\left(w_{11}, x_{10} y_{11}, z_{11}\right)-\left(w_{11}, x_{10}, z_{11} y_{11}\right)+$ $\left(w_{11}, x_{10}, y_{11}\right) z_{11}-\left(w_{11}, y_{11}, z_{11}\right) x_{10}=0$ or $\left(R_{11}, R_{11}, R_{11}\right) R_{10}=0$. Also, [ $\left(R_{11}\right.$, $\left.\left.R_{11}, R_{11}\right) R_{11}\right] R_{10} \subseteq\left(\left(R_{11}, R_{11}, R_{11}\right), R_{11}, R_{10}\right)+\left(R_{11}, R_{11}, R_{11}\right)\left(R_{11} R_{10}\right)=0$, using (iii) and earlier calculation that $\left(R_{11}, R_{11}, R_{10}\right)=0$. Hence $I_{1} R_{10}=0$. Then $I_{1}\left(R_{10} R_{01}\right) \subseteq\left(I_{1}, R_{10}, R_{01}\right)+\left(I_{1} R_{10}\right) R_{01}=0$, since we have shown previously $\left(R_{11}, R_{10}, R_{01}\right)=0$. From these last calculations and (iii), it now follows that $I_{1} H=0$. Similarly one may show $I_{0} H=0$.

Suppose $H=0$. Then $R_{10}=0=R_{01}$ implies $R_{11}$ and $R_{00}$ are ideals of $R$ such that $R_{11} R_{00}=0$. Since $R$ is a prime ring and $e \in R_{11}$, we must have $R_{00}=0$. But then $R=R_{11}$ implies $e=1$, contrary to assumption. Thus it must be the case $H \neq 0$. But $H \neq 0$ and $R$ a prime ring imply $I_{1}=0=I_{0}$, or that $R_{11}$ and $R_{00}$ are associative subrings of $R$.

We may now join the calculations on p. 316 of [2] to conclude that for any $x_{1}, x_{2}, x_{3} \in R$ one has $\left(x_{1}, x_{2}, x_{3}\right)=\operatorname{sgn} \sigma\left(x_{\sigma 1}, x_{\sigma 2}, x_{\sigma 3}\right)$ where $\sigma$ is any permutation of three elements. Since this implies $R$ is alternative, the proof is complete.

In conclusion, the author would like to express his appreciation to Professor Erwin Kleinfeld under whose direction this research was accomplished.

\section{BIBLIOGRAPHY}

1. A. A. Albert, Power-associative rings, Trans. Amer. Math. Soc. 64 (1948), 552-593. MR 10, 349.

2. E. Kleinfeld, Generalization of alternative rings. I, J. Algebra 18 (1971), 304-325. MR 43 \#308.

Department of Mathematics, University of Iowa, Iowa City, Iowa 52242 